\title{
Comunicação
}

\section{Carotenoides totais em resíduos do camarão Litopenaeus vannamei ${ }^{1}$}

\author{
Larissa Mont'Alverne Jucá Seabra², Karla Suzanne Florentino da Silva Chaves Damasceno ${ }^{3}$, \\ Camila Ricioli da Silva ${ }^{4}$, Camila de Carvalho Gomes ${ }^{4}$, Lucia Fátima Campos Pedrosa ${ }^{3}$
}

\section{RESUMO}

Considerando-se o possível aproveitamento dos resíduos, provenientes do beneficiamento do camarão, realizouse, neste trabalho, a determinação da concentração de carotenoides totais dos resíduos do camarão Litopenaeus vannamei e da farinha obtida após secagem. As análises foram realizadas nos resíduos in natura e na farinha recémprocessada (dia 0) e aos 60, 120 e 180 dias de armazenamento sob congelamento. Os resíduos frescos apresentaram, no dia 0 , teores de $42,74 \mu \mathrm{g} / \mathrm{g}$ de carotenoides totais e, a farinha recém-processada, de 98,51 $\mu \mathrm{g} / \mathrm{g}$. Após 180 dias de armazenamento, sob congelamento, os teores de carotenoides totais diminuíram significativamente, quando comparados com os do dia $0(p<0,05)$.

Palavras-chave: carotenoides, astaxantina, camarão, farinha.

\begin{abstract}
Total carotenoids in white shrimp (Litopenaeus vannamei) waste

Aiming at the utilization of shrimp processing wastes, this study carried out an analysis of total carotenoids in white shrimp (Litopenaeus vannamei) waste and in the dried shrimp waste meal. The analysis of total carotenoids was carried out in fresh wastes and processed flour (day 0) and after 60, 120 and 180 days of frozen storage. The fresh shrimp waste had $42.74 \mu \mathrm{g} / \mathrm{g}$ of total carotenoids and the dried meal $98.51 \mu \mathrm{g} / \mathrm{g}$. After 180 days of frozen storage, total carotenoids were significantly lower than day $0(p<0,05)$.
\end{abstract}

Key words: carotenoids, astaxanthin, shrimp, meal.

Recebido para publicação em 24/05/2011 e aprovado em 12/11/2013

${ }^{1}$ Extraído da Tese de Doutorado intitulada "Camarão Litopenaeus vannamei: componentes de importância nutricional na carne e na farinha obtida a partir dos resíduos do beneficiamento".

${ }^{2}$ Nutricionista, Doutora. Universidade Federal do Rio Grande do Norte, Avenida General Gustavo Cordeiro de Farias s/n, 59010-180, Natal, Rio Grande do Norte, Brasil. larissaseabra@ufrnet.br (autora para correspondência).

${ }^{3}$ Nutricionista, Doutora. Departamento de Nutrição, Universidade Federal do Rio Grande do Norte, Avenida General Gustavo Cordeiro de Farias s/n, 59010-180, Natal, Rio Grande do Norte, Brasil. karlasuzanne@ufrnet.br; lpedrosa@ufrnet.br

4 Nutricionista. Departamento de Nutrição, Universidade Federal do Rio Grande do Norte, Avenida General Gustavo Cordeiro de Farias s/n, 59010-180, Natal, Rio Grande do Norte, Brasil. metanutri@yahoo.com.br; camila.carvalhog@hotmail.com 


\section{INTRODUÇÃO}

O camarão branco do Pacífico (Litopenaeus vannamei) é uma importante espécie comercial, responsável por $90 \%$ da produção global de camarão cultivado (Nirmal \& Benjakul, 2009). No Brasil, as condições climáticas favoráveis e o domínio das tecnologias de produção transformaram o país em principal produtor de camarões das Américas (Poersch et al., 2006). Parte do camarão produzido é comercializada congelada, sem o cefalotórax, ou na forma de filé, gerando grande quantidade de resíduos, que muitas vezes são descartados incorretamente, podendo acarretar problemas ambientais.

Considerando-se que os resíduos do beneficiamento do camarão são constituídos de proteínas, lipídeos, minerais, quitina, além de serem fontes de carotenoides (Chakrabarti, 2002), seu reaproveitamento proporcionaria fonte alternativa de nutrientes para a alimentação animal ou, mesmo, humana, aumentando a produtividade do setor, além de contribuir para a diminuição do descarte clandestino no meio ambiente.

A astaxantina é o principal carotenoide encontrado em crustáceos (Niamnuy et al., 2008). Resultados encontrados por Sowmya \& Sachindra (2012) demonstraram a alta atividade antioxidante do extrato de astaxantina, proveniente de resíduos de camarão. Esse elevado poder antioxidante tem demonstrado efeitos benéficos em pacientes com câncer e hipertensão, aumentado assim o interesse por fontes de astaxantina (Hussein et al., 2005; Hix et al., 2005). Por causa do alto custo dos pigmentos sintéticos e da valorização dos produtos naturais, os resíduos produzidos durante o beneficiamento do camarão poderiam ser utilizados como fontes de pigmentos carotenoides em produtos alimentícios (Mandeville et al., 1992), ou em rações utilizadas na aquacultura (Liñán-Cabello \& Paniagua-Michel, 2004; Flores et al., 2007).

Dados referentes à concentração de carotenoides em resíduos de camarões, cultivados no Brasil, ainda são escassos. Desta forma, o objetivo deste trabalho foi determinar a concentração de carotenoides totais em resíduos e na farinha do camarão L. vannamei, ao longo do armazenamento sob congelamento.

\section{MATERIAL E MÉTODOS}

As amostras foram constituídas de três lotes do camarão L. vannamei, fornecidos por uma empresa localizada no Estado do Rio Grande do Norte, Brasil. Cada lote foi constituído de $20 \mathrm{~kg}$ de camarão fresco (80-90 camarões/ $\mathrm{kg}$ ), provenientes de três diferentes viveiros, os quais foram homogeneizados para uma maior representatividade da amostra. Os lotes foram obtidos no momento da despesca, colocados em caixa de isopor com gelo e transportados para o Laboratório de Análises de Alimentos do
Departamento de Nutrição da Universidade Federal do Rio Grande do Norte. Os camarões foram descascados no mesmo dia, para obtenção dos resíduos (cefalotórax, exoesqueleto e télsons) e da farinha utilizados nas análises. A farinha foi obtida por meio da secagem do resíduo, em estufa, a $70^{\circ} \mathrm{C}$, por oito horas, seguida de trituração em processador de alimentos doméstico, de acordo com Damasceno (2007). A porção muscular do camarão foi destinada à outra pesquisa, no mesmo laboratório.

A análise de carotenoides totais no tempo zero foi realizada no mesmo dia do recebimento. O restante do resíduo e da farinha foi armazenado sob congelamento $\left(-28,3^{\circ} \mathrm{C} \pm\right.$ $3,8)$, em embalagens individuais de polietileno $(1 \mathrm{~kg})$, e identificadas individualmente, de acordo com o tipo de amostra (resíduo ou farinha) e período de armazenamento (60, 120 e 180 dias). Antes da realização das análises, as embalagens contendo os resíduos foram colocadas sob refrigeração $\left(4{ }^{\circ} \mathrm{C} \pm 0,2\right)$ por 12 horas, quando então foi realizada sua trituração. As análises da farinha foram realizadas imediatamente após sua retirada do freezer. Cada amostra consistiu em porções de $1 \mathrm{~kg}$, que foram divididas aleatoriamente, para a realização das análises em triplicata. Com o intuito de caracterizar as amostras, foi realizada a análise da composição centesimal dos resíduos do camarão e da farinha, no dia 0 , sendo as determinações de umidade e de cinzas realizadas de acordo com Brasil (2005); de proteínas, de acordo com Bradford (1976), e do teor de lipídeos, segundo Bligh \& Dyer (1959). A extração dos carotenoides totais foi realizada de acordo com Sachindra et al. (2007), em 2,5 g das amostras de farinha e de resíduo, utilizando-se uma mistura de hexano e isoproponol (60:40). O cálculo da concentração de carotenoides, expresso em $\mu \mathrm{g}$ de astaxantina/g de amostra, foi realizado de acordo com a seguinte fórmula:

Carotenoides $(\mu \mathrm{g} / \mathrm{g})=\mathrm{A}_{468 \mathrm{~nm}} \times \mathrm{V}_{\text {extrato }} \times \mathrm{F} / 0,2 \times \mathrm{P}_{\text {amostra }}$

em que A é a absorbância, V é o volume do extrato e 0,2 é absorbância a $468 \mathrm{~nm}$ de $1 \mu \mathrm{g} / \mathrm{ml}$ de astaxantina padrão e Pé o peso da amostra, em gramas.

Para verificar diferenças nos valores de carotenoides totais, ao longo do período de armazenamento, realizouse Análise de Variância (ANOVA), seguida do teste de Duncan, a 5\% de significância. As análises estatísticas foram realizadas, utilizando-se o software "Statistica for windows" (Statsoft, 1997).

\section{RESULTADOS E DISCUSSÃO}

Com relação à composição química das amostras analisadas, foram encontrados valores percentuais de umidade, cinzas, lipídeos e proteínas de 73,7 $\pm 0,58 ; 5,0 \pm 0,14 ; 2,7 \pm$ 0,62 e $0,7 \pm 0,06$, para os resíduos, e de $8,9 \pm 0,23 ; 15,7 \pm 0,25$; $8,1 \pm 0,04$ e 11,8 $\pm 0,78$, para a farinha, respectivamente. 
As concentrações de carotenoides totais do resíduo fresco e da farinha de resíduos de camarão, neste estudo, foram de 42,74 e 98,51 $\mu \mathrm{g} / \mathrm{g}$, respectivamente (Tabela 1). Sachindra et al., 2007 encontraram concentração de 47,86 $\mu \mathrm{g} / \mathrm{g}$ de carotenoides totais, em resíduos de camarão da espécie Penaeus indicus.

Como a astaxantina é o principal carotenoide presente em crustáceos (Niamnuy et al., 2008), o aproveitamento dos resíduos do camarão em produtos alimentícios, além de contribuir para sua aceitação sensorial, poderia aumentar seu valor nutricional. Estudos sugerem que, para se obterem os efeitos benéficos da astaxantina, é necessário um consumo diário de cerca de $4 \mathrm{mg}$ deste carotenoide (Parisi et al., 2008; Satoh et al . 2009). As cápsulas de astaxantina comercializadas em alguns países apresentam concentração variando entre 4 e 20 mg (Seabra $\&$ Pedrosa, 2010). Considerando-se os valores obtidos neste estudo, em $100 \mathrm{~g}$ de resíduo ou de farinha de camarão, os teores de carotenoides totais corresponderam a 4,27 e 9,85 mg, respectivamente. Assim, estes produtos podem ser utilizados como aditivos naturais em alimento para uso animal ou humano.

Observa-se ainda, na Tabela 1, que a concentração de carotenoides totais diminuiu significativamente $(p<0,05)$ ao longo do período de armazenamento, tanto para os resíduos quanto para a farinha de camarão. Durante o armazenamento sob congelamento, geralmente, os carotenoides são preservados; no entanto, o descongelamento lento pode ser prejudicial. A exclusão de oxigênio, a proteção contra a luz, assim como a diminuição da temperatura diminuem a decomposição de carotenoides durante o armazenamento (Rodriguez-Amaya \& Kimura, 2004). Neste trabalho, os resíduos de camarão passaram 12 horas em temperatura de refrigeração, antes das análises; porém, a farinha foi analisada logo após sua retirada do freezer e apresentou maiores perdas de carotenoides.

Niamnuy et al. (2008) verificaram maior perda de astaxantina em camarões desidratados a $80^{\circ} \mathrm{C}$ do que nas amostras submetidas à desidratação em temperatura de $120^{\circ} \mathrm{C}$. Segundo os autores, em temperaturas mais elevadas ocorre a diminuição do tempo necessário para alcançar a umidade desejada para camarões desidratados, diminuindo assim a perda do pigmento, já que períodos de secagem mais longos resultam em maior tempo para a hidrólise da astaxantina esterificada, resultando em maior concentração de astaxantina livre que é mais susceptível à oxidação.

Neste estudo, tanto os resíduos na sua forma in natura quanto a farinha, obtida após a secagem, foram armazenados sob temperatura de congelamento, em embalagens plásticas, porém sem fechamento a vácuo, o que provavelmente contribuiu para a diminuição do teor de carotenoides.

Tabela 1 - Carotenoides totais $(\mu \mathrm{g} / \mathrm{g})$ de resíduos do camarão Litopennaeus vannamei e da farinha obtida após secagem, ao longo do congelamento por 180 dias

\begin{tabular}{lcccc}
\hline & \multicolumn{4}{c}{ Tempo (dias) } \\
\cline { 2 - 5 } & $\mathbf{0}$ & $\mathbf{6 0}$ & $\mathbf{1 2 0}$ & $\mathbf{1 8 0}$ \\
\hline Resíduo & $42,74^{\mathrm{a}} \pm 1,75$ & $36,63^{\mathrm{b}} \pm 0,21$ & $34,66^{\mathrm{bc}} \pm 0,83$ & $31,47^{\mathrm{c}} \pm 1,61$ \\
Farinha & $98,51^{\mathrm{a}} \pm 0,78$ & $80,00^{\mathrm{b}} \pm 0,27$ & $74,20^{\mathrm{c}} \pm 1,64$ & $65,41^{\mathrm{d}} \pm 1,13$ \\
\hline
\end{tabular}

$\mathrm{Na}$ horizontal, letras minúsculas iguais não diferem significativamente $(p>0,05)$.

\section{CONCLUSÕES}

Os resíduos do camarão L. vannamei e da farinha, obtidos após secagem, apresentam concentração considerável de carotenoides totais, que, entretanto, podem sofrer perdas ao longo do tempo de armazenamento. É importante a realização de estudos que utilizem condições especiais de armazenamento, com o intuito de diminuir a perda de carotenoides e de potencializar o uso desses produtos como fontes de astaxantina.

\section{REFERÊNCIAS}

Bligh G \& Dyer WJ (1959) A rapid method of total lipid extraction and purification. Canadian Journal of Biochemistry and Physiology, 37:911-917.

Bradford MM (1976) A rapid and sensitive method for the quantitation of microgram quantities of protein utilizing the principle of proteindye binding. Analytical Biochemistry, 72:248-54.
Brasil (2005) Ministério da Saúde. Agência Nacional de Vigilância Sanitária. Métodos Físico-Químicos para Análise de Alimentos. Edição IV. Brasília, Instituto Adolfo Lutz. 1020p.

Chakrabarti R (2002) Carotenoprotein from tropical brown shrimp shell waste by enzymatic process. Food Biotechnology, 16:81-90.

Damasceno KSFSC (2007) Farinha dos resíduos do camarão Litopenaeus vannamei: caracterização e utilização na formulação de hambúrguer. Tese de Doutorado. Universidade Federal de Pernambuco, Recife. 150p.

Flores M, Díaz F, Medina R, RE AD \& Licea A (2007) Physiological, metabolic and haematological responses in white shrimp Litopenaeus vannamei (Boone) juveniles fed diets supplemented with astaxanthin acclimated to low-salinity water. Aquaculture Research, 38:740-747.

Hix LM, Frey DA, Mclaws MD, Osterlie M, Lockwood SF \& Bertram JS (2005) Inhibition of chemically-induced neoplastic transformation by a novel tetrasodium diphosphate astaxanthin derivative. Carcinogenesis, 26:1634-1641. 
Hussein G, Nakamura M, Zhao Q, Iguchi T, Goto M, Sankawa V \& Watanabe H (2005) Antihypertensive and neuroprotective effects of astaxanthin in experimental animals. Biological and Pharmaceutical Bulletin, 28:47-52.

Liñán-Cabello MA \& Paniagua-Michel J (2004) Induction factors derived from carotenoids and vitamin A during the ovarian maturation of Litopenaeus vannamei. Aquaculture International, 12:583-592.

Mandeville S, Yaylayan V \& Simpon B (1992) GC/MS analysis of flavor-active compounds in cooked commercial shrimp waste. Journal of Agricultural and Food Chemistry, 40:1275-1279.

Niamnuy C, Devahastin S, Soponronnarit S \& Raghavan GSV (2008) Kinetics of astaxanthin degradation and color changes of dried shrimp during storage. Journal of Food Engineering, 87:591-600.

Nirmal NP \& Benjakul S (2009) Melanosis and quality changes of Pacific White Shrimp (Litopenaeus vannamei) treated with catechin during iced storage. Journal of Agricultural and Food Chemistry, 57:3578-3586.

Parisi V, Tedeschi M, Gallinaro G, Varano M, Saviano S \& Piermarocchi S (2008) Carotenoids and antioxidants in agerelated maculopathy Italian study. Ophthalmology, 115:324333
Poersch L, Cavalli RO, Wasielesky Júnior W, Castello JP \& Peixoto SRM (2006) Perspectivas para o desenvolvimento dos cultivos de camarões marinhos no estuário da Lagoa dos Patos, RS. Ciência Rural, 36:1337-1343.

Rodriguez-Amaya DB \& Kimura M (2004) Harvestplus Handbook for Carotenoid Analysis. Washington, IFPRI /CIAT. 63p.

Sachindra NM, Bhaskar N, Siddegowda GS, Sathisha AD \& Suresh PV (2007) Recovery of carotenoids from ensilaged shrimp waste. Bioresource Technology, 98:1642-1646.

Satoh A, Tsuji S, Okada Y, Murakami N, Urami M, Nakagawa K, Ishikura M, Katagiri M, Koga Y \& Shirasawa T (2009) Preliminary clinical evaluation of toxicity and efficacy of a new astaxanthin-rich Haematococcus pluvialis extract. Journal of Clinical Biochemistry and Nutrition, 44:280-284.

Statsoft (1997) Statistica for Windows. Tulsa, Statsoft Inc. CD ROM.

Seabra LMJ \& Pedrosa LFC (2010) Astaxanthin: Structural and functional aspects. Revista de Nutrição, 23:1041-1050.

Sowmya R \& Sachindra NM (2012) Evaluation of antioxidant activity of carotenoid extract from shrimp processing byproducts by in vitro assays and in membrane model system. Food Chemistry, 134:308-314. 